Original Article

\title{
Effect of oscillating electrical field stimulation on motor function recovery and myelin regeneration after spinal cord injury in rats
}

\author{
Da-Sheng Tian ${ }^{1)}$, Jue-HuA Jing $^{1)^{*}}$, Jun QIAN $^{1)}$, Lei Hen $^{1)}$, Bin Zhu $^{1)}$ \\ 1) Department of Orthopaedics, The Second Hospital of Anhui Medical University: Hefei 230601, \\ China
}

\begin{abstract}
Purpose] The aim of this study was to evaluate the effect of oscillating electrical field stimulation on motor function recovery and myelin regeneration in rats with spinal cord injury. [Subjects and Methods] A rat model of spinal cord injury was constructed by using the Allen weight-drop method. These rats were randomly divided into normal, spinal cord injury, and spinal cord injury + oscillating electrical field stimulation groups. The experimental group received the intervention with oscillating electrical field stimulation, and the control group received the intervention with an electrical field stimulator without oscillating electrical field stimulation. Each group was then randomly divided into seven subgroups according to observation time $(1,2,4,6,8,10$, and 12 weeks). Basso-Beattie-Bresnahan score and inclined plate test score evaluation, motor evoked potential detection, and histological observation were performed. [Results] In the first 2 weeks of oscillating electrical field stimulation, the oscillating electrical field stimulation and inclined plate test scores of spinal cord injury group and spinal cord injury + oscillating electrical field stimulation group were not significantly different. In the fourth week, the scores of the spinal cord injury group were significantly lower than those of the spinal cord injury + oscillating electrical field stimulation group. The motor evoked potential incubation period in the spinal cord injury + oscillating electrical field stimulation group at the various time points was shorter than that in the spinal cord injury group. In the sixth week, the relative area of myelin in the spinal cord injury + oscillating electrical field stimulation group was evidently larger than that in the spinal cord injury group. [Conclusion] Oscillating electrical field stimulation could effectively improve spinal cord conduction function and promote motor function recovery in rats with spinal cord injury, as well as promote myelin regeneration.

Key words: Oscillating electrical field, Spinal cord injury, BBB score
\end{abstract}

(This article was submitted Nov. 30, 2015, and was accepted Feb. 1, 2016)

\section{INTRODUCTION}

Several methods such as pharmacotherapy, surgical treatment, and physical therapy have been developed to treat spinal cord injury (SCI). In addition to these methods, electrical stimulation is an effective physical method used to treat $\mathrm{SCI}^{1-3)}$. Likewise, extra-low voltage field stimulation elicits curative effects for neural function recovery after SCI occurs ${ }^{4}$. Fehlings et al. ${ }^{5)}$ used an electrical field cathode or anode to stimulate the distal end of a SCI site and found that the regeneration ability of axons facing the cathode is stronger than that of axons facing the anode. Under electrical field stimulation, axons facing the anode for a long period can decrease in size ${ }^{6,7)}$. Therefore, the direction of an electrical field electrode greatly affects axon regeneration after SCI occurs ${ }^{8}$. To address the disadvantages of axonal growth caused by one-way electrical stimulation, researchers conducted oscillating electrical field stimulation (OFS). An OFS device can transform the polarity of an electrical field at an interval of $15 \mathrm{~min}$. As a result, cathode-directed neural axonal growth is promoted before anode rejection occurs;

*Corresponding author. Jue-Hua Jing (E-mail: dsdoccn@163.com)

(C2016 The Society of Physical Therapy Science. Published by IPEC Inc.

This is an open-access article distributed under the terms of the Creative Commons Attribution Non-Commercial No Derivatives (by-nc-nd) License $<$ http://creativecommons.org/licenses/by-nc-nd/4.0/>. 
thus, axons undergo a two-way growth. In 2000, OFS was permitted by the US Food and Drug Administration for treat of complete human SCI. In the same year, a clinical trial was initiated, in which all of the patients received decompression and methylprednisolone treatment before they participated in the clinical trial. In 2005, the results of this clinical trial revealed that treatment with OFS can improve human sensory and motor functions. Furthermore, It was found that this treatment mode is safe, reliable, and effective. Nevertheless, further studies should be conducted to determine whether OFS, or decompression and methylprednisolone treatment, improve nerve function. This study is based on the above-mentioned previous research.

In this study,the Basso-Beattie-Bresnahan (BBB) score, inclined plate test score, and motor evoked potential (MEP) of rats were determined in order to evaluate neural function recovery after OFS administration. A spinal cord tissue section was obtained to directly observe myelin regeneration $\left.{ }^{9}, 10\right)$.

\section{SUBJECTS AND METHODS}

One hundred and eighty healthy adult female Sprague-Dawley (SD) rats weighing $230 \pm 10 \mathrm{~g}$ were acquired from the Experimental Animal Centre of Anhui Medical University. This study was carried out in strict accordance with the recommendations in the Guide for the Care and Use of Laboratory Animals of the National Institutes of Health. The animal use protocol was reviewed and approved by the Institutional Animal Care and Use Committee (IACUC) of Anhui Medical University.

The SD rat model of SCI was established by using the Allen weight-drop method. The 180 SD rats were randomly divided into normal, SCI, and SCI + OFS groups. The normal group consisted of 12 rats, and the remaining groups consisted of 84 rats each. To successfully establish our model, we subjected hind legs and tail cramps to epidural congestion with an oscillating electrical field stimulator (Institute of Electrical Engineering, Chinese Academy of Sciences). In the experimental group (SCI+OFS group), an oscillating electrical field was applied as the intervention. In the control group (SCI group), the electrical field stimulator was applied without OFS as the interventions. The following parameters of OFS were set: current intensity of $40 \mu \mathrm{A}$, electrical field intensity on the spinal cord of $400 \mu \mathrm{V} / \mathrm{mm}$, and duration of one cycle of the oscillation period of $15 \mathrm{~min}$ (time until the electrical field polarity was changed). Power was supplied to the stimulator continuously, and the rats received continuous stimulation when they were awake. The current intensity of the stimulator was detected once every 3 days. The rats in each group were further divided randomly according to the following observation points: 1, 2, 4, 6 , 8,10 , and 12 weeks. Twelve rats were included in each subgroup. During the experiment, dead rats were replaced.

The initial weight of the rats was measured. Afterward, the rats were anesthetized with an intraperitoneal injection of $10 \%$ chloral hydrate $(0.3 \mathrm{ml} / 100 \mathrm{~g}$ of body weight $)$. The limbs and head of each rat were fixed with a tape on an operating table. The skin was then disinfected with $3 \%$ iodine solution. A longitudinal incision was made at the center of the T10 spinous process, along the midline of the back, with a length of approximately $2 \mathrm{~cm}$. The tissue layers were then cut open to reveal the T9-T11 laminae and spinous processes. The T9-T11 spinous processes were cut by using microscissors. The T10 lamina was carefully lifted to avoid tearing the ribs and damaging the blood vessels. The T10 spinal cord was subsequently exposed. The rats were then fixed on a New York University rat spinal cord weight-drop device (Shenzhen Ward). A 50-GCF potential impact was then induced by allowing $10 \mathrm{~g}$ of metal rods to fall freely from a height of $5 \mathrm{~cm}$ onto the spinal cord of the rats. The tails or hind legs of the rats twitched when their spinal cords were hit. After SCI was induced, the metal rods were removed immediately. After the SCI model was successfully established, two electrodes of the oscillating electrical field stimulator were fixed with unabsorbable sutures on both sides of the T9 and T12 spinous processes. The incision suture was then removed stepwise. The bodies of the rats were subjected to OSF in vitro. After the model was established, the experimental group was subjected to oscillating electrical field interference.

After SCI was induced, the rats received postoperative intraperitoneal injection of 100,000 units/day penicillin for 3 days to prevent infection. The rats with SCI were allowed to passively urinate two to three times per day until they established automatic micturition. In passive urination, the abdomen was lifted with the left hand, and the filled bladder was searched for with the right hand. Once located, the bladder was squeezed from the bottom part until urine discharged gradually. Once it was discharged completely, the perineum was kept dry to prevent infection.

Before or after modeling was completed at $1,2,4,6,8,10$, and 12 weeks, the rats in the SCI and SCI + OFS groups were scored in terms of BBB rating. The normal control rats were simultaneously scored.

BBB rating, which is one of the most commonly used assessment methods for rating the motor function of rats with SCI, is categorized into 21 levels. It is also used to observe hind limb activities, including the number and scope of the activity of each joint of the hind legs, the ability to support the body, and the coordination between the forelimb and hind limb. Furthermore, it can be divided into motion intensity in the early phase, coordination in the mid-phase, and fine activity in the late phase.

Two trained evaluators scored the experimental rats once a day based on BBB rating. The rats were placed on a large obstacle-free platform and then observed once for $5 \mathrm{~min}$. The average score was considered the final BBB rating.

Before or after modeling was completed at $1,2,4,6,8,10$, and 12 weeks, the rats in the SCI and SCI + OFS groups were subjected to the inclined plate test (Beijing Sport University). The normal control rats were also subjected to an inclined plate test.

In this test, the rats were positioned such that the body axis was along the longitudinal axis perpendicular to the inclined 
plate. Body balance was maintained on the inclined board by the strength of their forelimbs and hind limbs. In each repetition of the inclined plate test, the inclined plate was raised or lowered by $5^{\circ}$. The rat stayed in this position on the inclined plate for $5 \mathrm{sec}$, and this was considered the standard position. Furthermore, $5^{\circ}$ was set as the final value in the inclined plate test. To verify the authenticity of our test results, we ensured that the rats could maintain the same position on the inclined board for $5 \mathrm{sec}$. To construct the inclined plate, we used an inclined surface comprised of two plates, with one used as a bottom plate and the other used as a mobile inclined plate. A rubber pad with a thickness of $0.6 \mathrm{~cm}$ was fixed to the inclined surface. The side of the inclined plate was labeled with the corresponding angle for observation.

Two testers were trained. Each tester evaluated the experimental rats twice a day based on inclined plate test rating in the morning and afternoon. Average values were determined and recorded as the final results.

After the BBB and inclined surface test ratings were completed, the rats in all of the groups were anesthetized. MEPs were then detected by using a MEP instrument (Axon, USA). Two pairs of stimulating needle electrodes were placed near the gap between the T7/8 and L1/2 laminae. The positive electrode was directed to the anterior part, and the negative electrode was oriented to the posterior part. The distance between the two electrodes ranged from 0.5 to $0.8 \mathrm{~cm}$. The following parameters were set: stimulus type, rectangular pulse; constant voltage output; pulse width, $0.05 \mathrm{~ms}$; and output intensity, 100 to 150 V. A pair of recording electrodes was also used as needle electrodes. The electrodes were then placed on both sides of the gastrocnemius. Compound muscle action potentials were recorded as the final values. The latency difference between the anterior and posterior parts of the SCI site was considered as the final assessment result. A small difference indicated better recovery of nerve conduction function.

After $1,2,4,6,8,10$, and 12 weeks, the rats were anesthetized by intraperitoneally injecting $10 \%$ chloral hydrate. The chest was exposed, and the apex of the liver was continuously perfused with $0.9 \%$ normal saline until the color turned white. After the tissues were perfused with $4 \%$ paraformaldehyde for $30 \mathrm{~min}$ and fixed in $4 \%$ formalin for $12 \mathrm{~h}$, the injured spinal cord and spinal cord tissue located $1 \mathrm{~cm}$ near the end were removed. Slice were then embedded in optimum cooling temperature tissue freezing medium (Shanghai Wei Jin Biotech Co., Ltd., USA). Frozen sections were then laterally sliced with a thickness of $10 \mu \mathrm{M}$ by a cryostat (LEICA CM1850, Leica Microsystems Nussloch GmbH, Nussloch, Germany).

Luxol fast blue staining images were produced with Photoshop CS5, and the relative area of myelin was determined based on these images.

Data without specific notes are presented as mean \pm standard deviation (mean $\pm \mathrm{SD}$ ) values and they were analyzed by using SPSS version 10.0. A $\mathrm{p}<0.05$ was considered significantly different.

\section{RESULTS}

The BBB scores of the SCI and SCI+OFS group at different time points are shown in Table 1. In the first 2 weeks, the BBB score of the SCI+ OFS group was similar to that of the SCI group. In the fourth week, the BBB score of the OFS group was significantly higher than that of the SCI group $(\mathrm{p}<0.05)$. The BBB scores of the SCI and SCI+OFS groups also increased evidently between 2 and 6 weeks. Furthermore, the BBB scores gradually increased significantly after 6 weeks and remained almost constant in the last 2 weeks.

The inclined plate test scores at different time points are shown in Table 2. Although the inclined plate test scores of the SCI and SCI+OFS groups increased in the first 2 weeks of OSF, no significant difference was evident between the SCI and SCI+OFS groups. In the fourth week, the inclined plate test score of the SCI group was significantly lower than that of the SCI+OFS group $(p<0.05)$. The final inclined plate test score of the SCI+OFS group was significantly higher than that of the SCI group $(p<0.05)$. The inclined plate test score of the SCI and SCI+OFS groups also increased evidently between 2 and 6 weeks. Furthermore, the inclined plate test score increased gradually after 6 weeks and remained almost constant in the last 2 weeks.

The MEP test results of the SCI and SCI+OFS groups are shown in Table 3. The MEP values of the SCI+OFS group during each incubation week were lower than those of the SCI group. The MEP values of the two groups in the fourth week were significantly different $(\mathrm{p}<0.05)$. The initial and final MEP values in the same group were also significantly different $(p<0.05)$. Each MEP value remained stable in the last 2 weeks.

Luxol fast blue staining results are shown in Figs. 1 and 2, and Table 4. Myelin boundaries were relatively clear after the tissues were stained. One week after injury, no decrease in the stained area was evident when comparing the two treatment groups with the normal group after the tissues were analyzed with the image processing software. A sharp decrease was observed at 2 weeks. By contrast, an increase in the stained area was observed in the two groups after 6 weeks compared with the normal group. There were significant differences in these results between the SCI+OFS and SCI groups (p<0.05). Likewise, the final results were significantly different between these two groups.

\section{DISCUSSION}

SCI is a common clinical refractory disease that seriously harms human health. Studies on SCI treatment have been conducted, but no treatment specific for SCI has been developed yet ${ }^{11-13)}$. Nevertheless, electrical stimulation has been applied to promote spinal cord regeneration, repair SCI, and improve neural function recovery. 
Table 1. BBB detection values of rats in the SCI and SCI+OFS group at the different time points (unit: score)

\begin{tabular}{lcc}
\hline Time & $\begin{array}{c}\text { SCI group } \\
(\mathrm{n}=84)\end{array}$ & $\begin{array}{c}\text { SCI+OFS group } \\
(\mathrm{n}=84)\end{array}$ \\
\hline Before modeling & 21 & 21 \\
1st week & $2.3 \pm 1.2$ & $2.4 \pm 1.6$ \\
2nd week & $4.3 \pm 1.4$ & $4.6 \pm 1.5$ \\
4th week & $9.4 \pm 2.0$ & $11.8 \pm 2.6^{*}$ \\
6th week & $12.5 \pm 2.5$ & $16.5 \pm 3.4^{*}$ \\
8th week & $14.0 \pm 2.2$ & $17.5 \pm 3.1^{*}$ \\
10th week & $14.7 \pm 2.3$ & $17.7 \pm 3.2^{*}$ \\
12th week & $14.8 \pm 3.1$ & $17.8 \pm 3.8^{*}$ \\
\hline
\end{tabular}

Values are shown as the mean \pm standard deviation (SD). ${ }^{*} \mathrm{p}<0.05$
Table 2. Inclined plate test values of rats in the SCI and $\mathrm{SCI}+\mathrm{OFS}$ group at the different time points (unit: ${ }^{\circ}$ )

\begin{tabular}{lcc}
\hline Time & $\begin{array}{c}\text { SCI group } \\
(\mathrm{n}=84)\end{array}$ & $\begin{array}{c}\text { SCI+OFS group } \\
(\mathrm{n}=84)\end{array}$ \\
\hline Before modeling & $63.4 \pm 4.8$ & $64.1 \pm 5.1$ \\
1st week & $10.8 \pm 2.0$ & $11.2 \pm 1.5$ \\
2nd week & $14.5 \pm 2.2$ & $16.4 \pm 3.8$ \\
4th week & $30.5 \pm 3.8$ & $37.2 \pm 4.9^{*}$ \\
6th week & $38.5 \pm 5.2$ & $45.4 \pm 6.2^{*}$ \\
8th week & $43.3 \pm 5.8$ & $52.2 \pm 5.4^{*}$ \\
10th week & $46.4 \pm 5.0$ & $55.3 \pm 6.1^{*}$ \\
12th week & $47.9 \pm 5.6$ & $57.8 \pm 6.4^{*}$ \\
\hline
\end{tabular}

Values areshown as the mean \pm standard deviation (SD). ${ }^{*} \mathrm{p}<0.05$
Table 3. MEP latency values of rats in the SCI and SCI+OFS group at the different time points (unit: $\mathrm{ms}$ )

\begin{tabular}{lcc}
\hline Time & $\begin{array}{c}\text { SCI group } \\
(\mathrm{n}=84)\end{array}$ & $\begin{array}{c}\text { SCI }+ \text { OFS group } \\
(\mathrm{n}=84)\end{array}$ \\
\hline Before modeling & $3.91 \pm 0.14$ & $3.99 \pm 0.17$ \\
1st week & $12.01 \pm 0.55$ & $11.62 \pm 0.62$ \\
2nd week & $10.38 \pm 0.64$ & $9.83 \pm 0.68$ \\
4th week & $9.45 \pm 0.75$ & $7.94 \pm 0.50^{*}$ \\
6th week & $8.73 \pm 0.41$ & $6.62 \pm 0.32^{*}$ \\
8th week & $8.14 \pm 0.55$ & $5.86 \pm 0.23^{*}$ \\
10th week & $7.62 \pm 0.51$ & $5.46 \pm 0.27^{*}$ \\
12th week & $7.48 \pm 0.82$ & $5.32 \pm 0.44^{*}$ \\
\hline
\end{tabular}

Table 4. Myelin area ratios in images of tissue sections in the SCI and SCI + OFS groups at the different time points

\begin{tabular}{lcc}
\hline Time & $\begin{array}{c}\text { SCI group } \\
(\mathrm{n}=84)\end{array}$ & $\begin{array}{c}\text { SCI+OFS group } \\
(\mathrm{n}=84)\end{array}$ \\
\hline 1st week & $60.4 \pm 6.6$ & $61.2 \pm 7.8$ \\
2nd week & $41.3 \pm 3.9$ & $42.9 \pm 3.8$ \\
4th week & $42.9 \pm 4.2$ & $45.3 \pm 3.7$ \\
6th week & $44.5 \pm 5.2$ & $55.7 \pm 6.4^{*}$ \\
8th week & $45.5 \pm 4.7$ & $61.2 \pm 6.9^{*}$ \\
10th wee & $49.3 \pm 6.3$ & $63.6 \pm 7.2^{*}$ \\
12th week & $50.1 \pm 6.5$ & $65.2 \pm 9.0^{*}$ \\
\hline
\end{tabular}

Values are shown as the mean \pm standard deviation (SD). ${ }^{*} \mathrm{p}<0.05$

Values are shown as the mean \pm standard deviation (SD). *p $<0.05$

Nerve regeneration in the spinal cord is promoted by stimulation with an electrical field in several modes, including direct current field, pulsed electrical field, alternating current field, and oscillating electrical field. Electric stimulation technologies can promote spinal cord regeneration. Experimental results have shown that regeneration occurs at a rapid rate when axons are oriented toward an electrical field cathode. By contrast, degeneration occurs when axons are directed toward an electrical field anode ${ }^{14)}$. Further studies showed that oscillating electrical field can promote nerve regeneration and functional recovery of the spinal cord, and exhibit biologically safety. Indeed, electrical field stimulation provides a new direction for the treatment of SCI. In our research, an oscillating electrical field was applied based on previous experimental results.

An objective evaluation method is necessary to promote the recovery of neural function after SCI occurs. Common methods used to evaluate function and tissue recovery include behavioral assessment, histological analysis, and electrophysiological monitoring, among others. Accurate, reliable, and suitable methods should be selected to assess the success of function and tissue recovery after $\mathrm{SCI}$ occurs ${ }^{15}$.

BBB rating is the most commonly used method to assess motor function after SCI occurs ${ }^{9}$. This method was designed by Basso, Beattie, and Bresnahan to evaluate the functional recovery of the two hind legs after thoracic SCI occurred in rats. BBB rating is widely used because it can provide reliable and repeatable results that provide relevant data. Furthermore, BBB ratings can be interpreted easily, and behavioral changes can be quantified. Special training is not necessary to evaluate animals $^{16}$. However, BBB rating is limited because it depends on the observer's subjectivity ${ }^{16}$. To reduce errors due to subjective judgment, two separate and trained assessors were used to verify the accuracy of evaluations in the present study.

In this study, the experimental group received an intervention with an oscillating electrical field (OFS) immediately after SCI occurred, and the control group was exposed to the oscillating electric stimulator without OFS. The BBB scores indicated that the two groups did not show improvement in the first 2 weeks. After 4 weeks, oscillating electrical fields promoted the motor function of the hind limbs. After 4 to 6 weeks, the BBB scores of the experimental group increased faster than those of the control group. After 10 weeks, The BBB score increased gradually. After 4 more weeks, the BBB score of the experimental group was significantly higher than that of the control group. By contrast, the BBB score of the control group was gradually and consistently restored as time was extended. This result is consistent with that described in previous research ${ }^{17)}$. Thus, OFS can repair SCI and improve neurological function recovery after SCI in the hind legs of rats. 


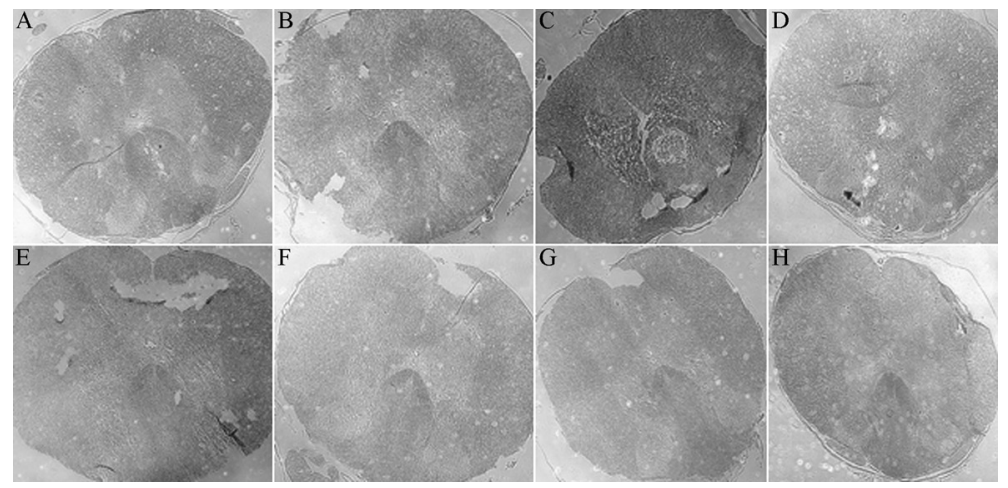

Fig. 1. The spinal cord pathology of rats in the normal group and spinal cord injury + oscillating electrical field stimulation group at each time point (solid blue stain, $40 \times$ )

A: The spinal cord pathology of the normal rat; B-H: The spinal cord pathology of rats after spinal cord injury ( $1 \mathrm{w}, 2 \mathrm{w}, 4 \mathrm{w}, 6 \mathrm{w}, 8 \mathrm{w}, 10 \mathrm{w}$, and $12 \mathrm{w}$, respectively).

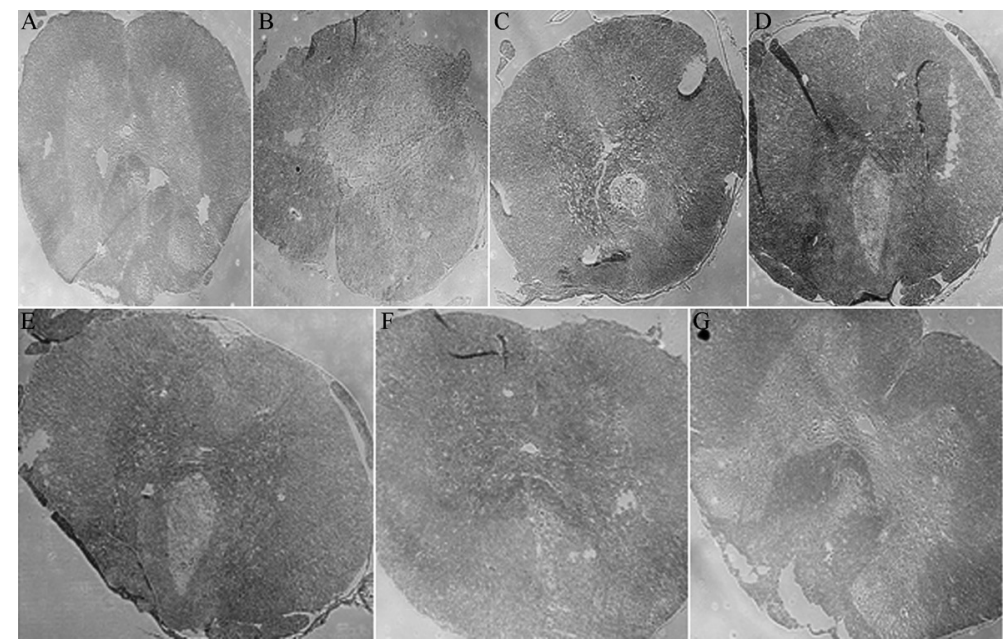

Fig. 2. The spinal cord pathological conditions of rats in the spinal cord injury group at each time point (solid blue stain, $40 \times$ )

A-G: Pathological conditions of the rats after spinal cord injury $(1 \mathrm{w}, 2 \mathrm{w}, 4 \mathrm{w}, 6 \mathrm{w}, 8 \mathrm{w}, 10 \mathrm{w}$, and 12 w, respectively).

In an inclined plate test evaluation, which was initially reported in 1977 by Rivlin and Tator ${ }^{18)}$, a cantboard is used to detect neural function recovery after SCI in rats. Fehlings et al. ${ }^{19)}$ indicated that this method requires simple equipment. This simple, rapid, and noninvasive method exhibits good repeatability and a high degree of correlation between SCI cases. Therefore, it can be used as an evaluation index of SCI. In this study, two methods, namely the BBB score and inclined plate test score, were used to quantify rat behavior. These two methods revealed similar results, indicating that both methods exhibit good repeatability and operability.

MEP is applied to stimulate the brain motor area or the efferent pathway by electrical or magnetic cues, excitatory junction potentials, and excitation signals transmitted posteriorly via the extrapyramidal cone system in the spinal cord. As a result, depolarization is induced in anterior horn cells or peripheral nerve fibers of the spinal cord. The electrical response corresponding to the reaction in the distal spinal cord and peripheral nerve or muscle is then recorded. The recorded electrical response is considered as MEP. The latent period and amplitude of MEP can directly reflect the functional status of descending tracts and the peripheral motor nerves of the spinal cord. This parameter is an important objective measure of motor function recovery after SCI occurs ${ }^{20)}$. Furthermore, the MEP incubation period can indicate the nerve conduction velocity ${ }^{21,22)}$. The incubation period can also accurately show whether spinal movement function is absent or altered. Amplitude is also one of the main MEP indices observed, and it can objectively reflect axonal damage. However, the MEP amplitude varies between individuals and must be determined a large number of times in each individual, and its quantitative credibility is poor. Therefore, the MEP amplitude was not included in our experimental observations. 
This study suggests that the motor function of both hind limbs after induced SCI did not recover within 2 weeks in the SCI+OFS group. The MEP incubation period was shortened, but no statistical significance was observed between the experimental and control groups. This difference may have been observed because glial scar formation did not occur in 2 weeks. Early intervention with OFS increases the speed of axonal growth as electrophysiological changes occur, but this rapid axonal growth is insufficient to result in motor function recovery. After 4 weeks, the incubation period of the two groups was shortened. The MEP values of the SCI+OFS group were significantly different from those of the control group. After 10 weeks, the MEP value increased gradually.

Histological observation was performed by biopsy staining to observe myelin regeneration of the spinal cord. Myelin is a fat tissue layer that covers axons to provide insulation. This covering also improves the speed of nerve impulse transmission and protects the axons in the central and peripheral nervous systems. The area of the myelin sheath can be measured in a cross section by staining and by using image processing software. For instance, solid blue dye is a copper-phthalic compound with staining properties. This dye binds to myelin phospholipids in ethanol solution. After staining, we can observe that myelin appears brightly colored. Solid blue staining represents specific staining and thus is used as an efficient marker to identify myelin ${ }^{23)}$. It has been applied to observe changes in myelin, such as demyelinating lesions and myelin regeneration. Solid blue staining has also been performed to observe the distribution of positive Luxol fast blue-stained fibers ${ }^{24-26)}$.

In this study, solid blue staining was conducted to evaluate SCI. Our results showed that myelin boundaries were relatively cleared after staining was completed. Image processing analysis showed no evident decrease in the area of myelin boundaries in the two groups compared with the normal group 1 week after SCI. A sharp decrease was then observed after 2 weeks. By contrast, an increase in the stained area was observed in the SCI and SCI + OFS groups after 6 weeks compared with the normal group. There were significant differences in these results between the SCI+OFS and control groups. A significant difference was also observed in the final results of these two groups. Therefore, the neural functional recovery of the SCI+OFS group was more efficient than that of the SCI group. Indeed, our hypothesis was verified. Behavioral assessment and electrophysiological monitoring results were consistent. However, the histological examination results were significantly different from the behavioral assessment results and electrophysiological detection. This difference could be attributed to the lack of immediate function after the injury occurred. Sufficient time may also have been necessary to allow the myelin sheath to fully disintegrate. Although the results of the histological examination were different from those of behavioral assessment and electrophysiological detection, OFS promoted myelin regeneration after induction of SCI in the rats in this study.

In summary, OFS can effectively promote motor function recovery of rats with SCI. More than 4 weeks were necessary to promote neural functional recovery of the rats with SCI by OFS, although the effect of OFS was significantly weakened after 10 weeks. Furthermore, OFS can promote myelin regeneration.

\section{REFERENCES}

1) Cho HY, Kim EH, Kim B, et al.: Effects of repetitive high frequency transcutaneous electrical nerve stimulation (HF-TENS) onspasticity and motor function following spinal cord injury in rats. J Phys Ther Sci, 2012, 24: 133-137. [CrossRef]

2) Fehlings MG, Rabin D, Sears W, et al.: Current practice in the timing of surgical intervention in spinal cord injury. Spine, 2010, 35: S166-S173. [Medline] [CrossRef]

3) Bi X, Lv H, Chen BL, et al.: Effects of transcutaneous electrical nerve stimulation on pain in patients with spinal cord injury: a randomized controlled trial. J Phys Ther Sci, 2015, 27: 23-25. [Medline] [CrossRef]

4) Fehlings MG, Tator CH: The effect of direct current field polarity on recovery after acute experimental spinal cord injury. Brain Res, 1992, 579: 32-42. [Medline] [CrossRef]

5) Fehlings MG, Tator CH, Linden RD: The effect of direct-current field on recovery from experimental spinal cord injury. J Neurosurg, 1988 , 68: 781-792. [Medline] [CrossRef]

6) McCaig CD: Spinal neurite reabsorption and regrowth in vitro depend on the polarity of an applied electric field. Development, 1987, 100: 31-41. [Medline]

7) Hamid S, Hayek R: Role of electrical stimulation for rehabilitation and regeneration after spinal cord injury: an overview. Eur Spine J, 2008 , 17: 1256-1269. [Medline] [CrossRef]

8) Hernández-Labrado GR, Polo JL, López-Dolado E, et al.: Spinal cord direct current stimulation: finite element analysis of the electric field and current density. Med Biol Eng Comput, 2011, 49: 417-429. [Medline] [CrossRef]

9) Basso DM, Beattie MS, Bresnahan JC: A sensitive and reliable locomotor rating scale for open field testing in rats. J Neurotrauma, 1995, 12: 1-21. [Medline] [CrossRef]

10) Shinozaki M, Yasuda A, Nori S, et al.: Novel method for analyzing locomotor ability after spinal cord injury in rats: technical note. Neurol Med Chir (Tokyo), 2013, 53: 907-913. [Medline] [CrossRef]

11) Suyama T, Takahashi K, Shibuta H, et al.: Pain and rehabilitation in patients with spinal cord injury. J Phys Ther Sci, 2001, 13: 59-64. [CrossRef]

12) Wilcox JT, Cadotte D, Fehlings MG: Spinal cord clinical trials and the role for bioengineering. Neurosci Lett, 2012, 519: 93-102. [Medline] [CrossRef]

13) Kim JE, Chung YJ, Shin HK: Effects of balance training on patients with spinal cord injury. J Phys Ther Sci, 2010, 22: 311-316. [CrossRef]

14) Shapiro S, Borgens R, Pascuzzi R, et al.: Oscillating field stimulation for complete spinal cord injury in humans: a phase 1 trial. J Neurosurg Spine, 2005, 2: 3-10. [Medline] [CrossRef]

15) Milicevic S, Piscevic V, Bukumiric Z, et al.: Analysis of the factors influencing functional outcomes in patients with spinal cord injury. J Phys Ther Sci, 2014, 26: 67-71. [Medline] [CrossRef] 
16) Metz GA, Merkler D, Dietz V, et al.: Efficient testing of motor function in spinal cord injured rats. Brain Res, 2000, 883: 165-177. [Medline] [CrossRef]

17) You SW, Chen BY, Liu HL, et al.: Spontaneous recovery of locomotion induced by remaining fibers after spinal cord transection in adult rats. Restor Neurol Neurosci, 2003, 21: 39-45. [Medline]

18) Rivlin AS, Tator CH: Objective clinical assessment of motor function after experimental spinal cord injury in the rat. J Neurosurg, 1977, 47: 577-581. [Medline] [CrossRef]

19) Fehlings MG, Tator $\mathrm{CH}$ : The relationships among the severity of spinal cord injury, residual neurological function, axon counts, and counts of retrogradely labeled neurons after experimental spinal cord injury. Exp Neurol, 1995, 132: 220-228. [Medline] [CrossRef]

20) Agrawal G, Iyer S, All AH: A comparative study of recording procedures for motor evoked potential signals. Conf Proc IEEE Eng Med Biol Soc, 2009, 2009: 2086-2089.

21) Hilibrand AS, Schwartz DM, Sethuraman V, et al.: Comparison of transcranial electric motor and somatosensory evoked potential monitoring during cervical spine surgery. J Bone Joint Surg Am, 2004, 86-A: 1248-1253. [Medline]

22) Kim SR, Kwon KH, Cho BJ: The effects of neuromuscular electrical stimulation on pharyngeal transit time. J Phys Ther Sci, 2013, 25: 849-851. [Medline] [CrossRef]

23) Deloire-Grassin MS, Brochet B, Quesson B, et al.: In vivo evaluation of remyelination in rat brain by magnetization transfer imaging. J Neurol Sci, 2000, 178: 10-16. [Medline] [CrossRef]

24) Sun SW, Liang HF, Trinkaus K, et al.: Noninvasive detection of cuprizone induced axonal damage and demyelination in the mouse corpus callosum. Magn Reson Med, 2006, 55: 302-308. [Medline] [CrossRef]

25) Tyor WR, Avgeropoulos N, Ohlandt G, et al.: Treatment of spinal cord impact injury in the rat with transforming growth factor-beta. J Neurol Sci, 2002, 200: 33-41. [Medline] [CrossRef]

26) Sribnick EA, Wingrave JM, Matzelle DD, et al.: Estrogen attenuated markers of inflammation and decreased lesion volume in acute spinal cord injury in rats. J Neurosci Res, 2005, 82: 283-293. [Medline] [CrossRef] 